\title{
Cuore-Fegato-Rene: quando l'unione non fa la forza
}

\author{
G. Ferro ${ }^{1}$ e M. Masini ${ }^{2}$ \\ ${ }^{1}$ UO Nefrologia e Dialisi \\ ${ }^{2}$ Cardiologia, Ospedale Santa Maria Annunziata, Azienda Sanitaria di Firenze
}

\section{Il caso clinico}

È qui descritto il caso di un uomo di 48 anni di nazionalità marocchina, ex fumatore, non bevitore con familiarità per diabete mellito (entrambi i genitori e 1 fratello). All'età di 26 anni è riscontrato diabete mellito per il quale non pratica alcuna terapia. Giunto in Italia nel 2002 (41 anni) esegue una visita diabetologica in cui è riscontrato grave scompenso glico-metabolico (glicemia $300 \mathrm{mg} / \mathrm{dL}, \mathrm{Hb}$ glicata 12.1\%), insufficienza renale (creatinina $1.2 \mathrm{mg} / \mathrm{dL}$ ), retinopatia diabetica avanzata, neuropatia periferica, ipertensione arteriosa (PA 150/90 mmHg). Il paziente inizia terapia insulinica ed antipertensiva ma con scarsa aderenza sia alla dieta che alla terapia. Negli anni successivi nei pochi esami di controllo effettuati si assiste ad un progressivo peggioramento della funzione renale con comparsa di proteinuria nefrosica e pertanto è indirizzato a un Reparto nefrologico. Il paziente giunge nel 2007 alla nostra osservazione in condizioni cliniche precarie lamentando malessere generale, anoressia, astenia intensa e riferendo episodi di vomito alimentare con progressiva riduzione del peso corporeo. $\grave{E}$ riscontrata grave ipertensione arteriosa $(190 / 90 \mathrm{mmHg})$ ed un quadro laboratoristico (Tab. I) di scompenso metabolico, insufficienza renale cronica avanzata con proteinuria nefrosica, anemia normocitica-normocromica. Non presenti segni clinici rilevanti di scompenso cardiaco (classe I-II NYHA). Il paziente effettua screening immunologico ed infettivologico (anche per ricerca BK) con esito negativo. Per l'evidenza di anemia refrattaria alla terapia con eritropoietina e vista l'assenza di sangue occulto nelle feci, della normalità degli indici di emolisi, degli indici di flogosi e dei marcatori neoplastici, effettua BOM che risulta nei limiti della norma. Si evidenzia incremento degli indici di colestasi senza iperbilirubinemia e con un quadro ecografico epatico normale. Dal punto di vista ecocardiografico (Tab. II) non particolari rilievi patologici se non iniziale ipertensione polmonare, lieve insufficienza mitralica e tricuspidalica.

Nel corso dei mesi successivi pur ottimizzando la terapia antipertensiva e per il diabete si assiste al rapido e progressivo peggioramento delle condizioni generali con l'insorgenza di un quadro di scompenso cardiaco conclamato (classe IV NYHA) ed evidenza ecocardiografica di grave scompenso diastolico (Tab. II) con ipertensione polmonare severa, al punto che il paziente è ricoverato più volte con quadro di edema polmonare ed anasarca. Viene deciso di eseguire coronarografia che esclude lesioni coronariche. Per il peggioramento contestuale della funzione renale il paziente inizia terapia sostitutiva della funzione renale mediante emodialisi. L'inizio della terapia dialitica consente di correggere le alterazioni correlate all'uremia ma non migliora i sintomi ascrivibili allo scompenso cardiaco nonostante la rimozione di liquidi (riduzione del peso corporeo di $8 \mathrm{~kg}$ ), anzi addirittura si assiste ad un peggioramento della sintomatologia con comparsa di epatopatia a carattere colestatico per incremento degli enzimi di colestasi ma con transaminasi solo lievemente aumentate, bilirubina e quadro ecografico nella norma.

A distanza di un anno dall'inizio della dialisi il paziente continua ad avere un grave scompenso cardiaco (classe III-IV NYHA) con incremento persistente degli indici di colestasi.

\section{Discussione}

In questo caso clinico le problematiche non chiarite sono: 
TABELLA I - ANDAMENTO DEI PRINCIPALI ESAMI DI LABORATORIO

\begin{tabular}{lllll}
\hline & Marzo 2007 & Luglio 2007 & $\begin{array}{l}\text { Gennaio 2008 } \\
\text { (inizio emodialisi) }\end{array}$ & Marzo 2008 \\
\hline s.creat mg/dL & 3.6 & 5.7 & 6,5 & Dialisi \\
$\mathrm{Hb}$ g/dL & 9.2 & 8.7 & 7 & 12 \\
Pcr mg/dL & 0.4 & 0.7 & 2 & 1 \\
Alb g/dL & 3.5 & 3.4 & 3 & 3.5 \\
F.Alc. U/I & 170 & 257 & 103 & 209 \\
GGT U/1 & 110 & 261 & 64 & 180 \\
Got/Gpt UI/1 & $23 / 25$ & $17 / 20$ & $10 / 6$ & $13 / 11$ \\
Hb Glic \% & 12.5 & 8.5 & 9 & 6 \\
\hline
\end{tabular}

TABELLA II - ANDAMENTO DEI PRINCIPALI PARAMETRI ECOCARDIOGRAFICI

\begin{tabular}{lllll}
\hline & Marzo 2007 & Luglio 2007 & $\begin{array}{l}\text { Gennaio 2008 } \\
\text { (inizio emodialisi) }\end{array}$ & Marzo 2008 \\
\hline V sin DD/DS mm & $47 / 31$ & $51 / 34$ & $48 / 32$ & $46 / 32$ \\
SIV/PP mm & $12 / 11$ & $12 / 12$ & $15 / 12$ & $13 / 13$ \\
A sin mm & 36 & 36 & 39 & 38 \\
D\%/FE\% & $34 / 60$ & $33 /$ & $33 / 60$ & 30 \\
Pattern diastolico & Normale & Normale & Pseudonormale & Restrittivo \\
Ipert. Polm. & Moderata & Lieve & Severa & Severa \\
\hline
\end{tabular}

- Qual è la causa dello scompenso cardiaco?

- Come mai l'inizio dell'emodialisi con la sottrazione di liquidi e la correzione dei sintomi legati all'uremia non migliora il quadro clinico, che anzi peggiora?

- Qual è la causa della patologia epatica dal momento che è stata esclusa una patologia ostruttiva e/o infettiva?

Lo scompenso cardiaco di cui soffre il paziente è dal punto di vista ecocardiografico prevalentemente di tipo diastolico. La "disfunzione diastolica" è definita come una incapacità dei ventricoli a distendersi indipendentemente dalla capacità contrattile e quindi dalla frazione di eiezione FE (1). Infatti, nel 35\% dei pazienti con diagnosi di scompenso cardiaco, la funzione sistolica è normale e la disfunzione diastolica precede quella sistolica (1).

La disfunzione diastolica può evolvere fino a diventare una vera e propria patologia restrittiva in grado di determinare congestione venosa ed edema polmonare configurando un quadro di scompenso cardiaco vero e proprio (1). Questa evoluzione può essere ben caratterizzata e quantificata all'ecocardiogramma.

Nel nostro paziente sono state escluse gran parte delle patologie che entrano in diagnosi differenziale: ischemia miocardica, malattie infiltrative, patologie valvolari cardiache primitive, pericardite costrittiva, stati ipercinetici come tireotossicosi, malattie polmonari croniche con ipertensione polmonare (2).

La terapia ottimale dello scompenso diastolico prevede secondo linee guida internazionali (ACC/AHA 2005) ed in base ai livelli di evidenza:

- Controllo farmacologico dell'ipertensione arteriosa (IA) e della frequenza cardiaca se presente fibrillazione atriale (IC).

- Terapia diuretica per il sovraccarico idrico (IC).

- Rivascolarizzazione coronarica (IIa-C).

- Ripristino e mantenimento del Ritmo Sinusale se Fibrillazione Atriale (IIb-C).

- Uso di beta-bloccanti, ACE-i; sartanici, calcio antagonisti per il controllo dei sintomi (IIb-C).

- Incerto l'uso della digitale per il controllo dei sintomi (IIb-C). 


\section{L'ipotesi unificante}

Ci sembra importante rilevare che come momento patogenetico unificante potrebbe essere identificato il diabete mellito scompensato che può determinare un quadro di glicogenosi epatica legata ad accumulo di AGE e che correla con disfunzione cardiaca di tipo diastolico (3-5). Inoltre, ha sicuramente contribuito alla gravità del quadro clinico, il cosiddetto fenomeno del "Late referral" genericamente inteso come l'accesso tardivo ad una diagnosi e quindi ad una cura appropriata e che in questo caso ha riguardato sia il diabete mellito ma anche l'insufficienza renale.

\section{Indirizzo degli Autori:}

G. Ferro, MD

UO Nefrologia e Dialisi

Ospedale Santa Maria Annunziata

Via dell'Antella, 58

50011 Firenze

giuseppe.ferro@asf.toscana.it

\section{Bibliografia}

1. Aurigemma GP. Clinical Practice. Diastolic heart failure. N Engl J Med 2004; 351: 1097-105.

2. Guha A, Harmancey R, Taegtmeyer. Non ischemic heart failure in diabetes mellitus. Curr Opin Cardiol 2008; 23: 241-8.

3. Olsson R, Wesslau C, William-Olsson T, et al. Elevated aminotransferases and alcaline phosphates in unstable diabetes mellitus without ketoacidosis or hypoglycemia. J Gastroent 1989, 11: 541-5.

4. Munns CF, McCrossin RB, Thomsett MJ, et al. Hepatic glycogenosis reversible hepatomegaly in type 1 diabetes .J Paediatr 2000; 36: 449-52.

5. Toberson M, Chen YY, Brunt E, et al. Glycogenic hepatopathy: am underrecognized hepatic complication of diabetes mellitus . Am J Surg Pathol 2006; 30: 508-13.

6. Hunt SA, Abraham WT, Chin MH, et al. ACC/AHA 2005 Guidline Update for the Diagnosis and Managment of chronic heart failure in the adult. Circulation 2005; 112:154-235.

\section{TEST di VERIFICA - 5}

1) Quale tra questi fattori è maggiormente implicato nella patogenesi dello scompenso cardiaco diastolico:

a) Ipertensione arteriosa

b) Diabete mellito

c) Insufficienza renale

d) Tutte le precedenti

2) Quale tra questi fattori non caratterizza lo scompenso cardiaco diastolico:

a) Ventricolo sinistro dilatato

b) FE normale

c) Dilatazione della vena cava superiore

3) L'epatopatia correlata al diabete mellito scompensato è caratterizzata da:

a) Steatosi

b) Glicogenosi

c) Entrambe

Le risposte corrette alle domande sono a pag. 53 